\title{
INCIDENCE DE L'IMPLANTATION ET DE LA DIFFUSION DES CULTURES INDUSTRIELLES SUR L'ALIMENTATION DU BETAIL AU CAMEROUN
}

\author{
E. ENGUELEGUELE
}

\section{RESUME}

La création au Cameroun d'un élevage moderne est décidée. Céréales et racines existent mais sont consommées par l'homme, La culture du coton s'est largement développée dans le Nord. La production annuelle de tourteau pourrait atteindre 35000 tonnes. La canne a sucre alimente la sucrerie de MBandjock, aupres de 35000 tonnes. La canne a sucre alimente la sucrerie de MBandjock, aupres de
laquelle un centre d'embouche pourrait être créé. La culture du blé est possiblo dans le Nord et l'Adamaoua. Elle pourrait donner des sons pour l'embouche. La politique de l'élevage visera d'abord à utiliser ces sous-produits disponibles plutôt que de les exporter. Des campagnes de complementation alimentaire de saison sèche vont être entreprises. Des coopératives d'emboucheurs seront créées. Un essai de complémentation de saison sèche est décrit : il s'est révélé profitable.

\section{SUMMARY}

Effects of the establishment and spreading of industrial crops on cattle feed in the Cameroon

It has been decided to create modern husbandry in the Cameroon. Cereals and roots do exist but are consumed by man. Cotton growing has broadly developed in the North. The annual cattle cake production might reach 35,000 tons. Sugar cane supplies the MBandjock sugar factory, near which a fattening centre could be created. Wheat growing is possible in the North and the Adamaoua. This could provide bran for fattening. The livestock policy is firstly aimed at using these available by-products rather than exporting them. Supplemental feed
campaigns in the dry season will be undertaken. Fattering co-operative societies will be created. A supplemental feed test in the dry season is described - it is proved to be profitable.

\section{1. - POSITION DU PROBLEME}

" Notre action s'orientera vers l'amélioration de la qualité de l'élevage traditionnel et la création d'un secteur moderne d'élevage intensif."

Le cadre dans lequel devaient être menées les recherches zootechniques au cours du III' Plan Quin. quennal de Développement Economique et Social du Cameroun, était ainsi défini en 1971 par le Chef de l'Etat.

Si la plupart des problèmes posés par l'avènement de ce secteur moderne d'élevage intensif ont été effleurés et dégrossis dans nos laboratoires, celui ayant trait à l'alimentation occupera pendant de nombreuses années encore, les techniciens ceuvrant pour la promotion de notre élevage. Ceci donne la mesure du problème C'est que, sous les tropiques, d'une façon générale, le menu présenté classiquement en pareil cas est commun à l'animal et à l'homme, à une époque où le spectre de la faim dans le tiers monde angoisse ceux qui ont la lourde mission de planifier les ressources alimentaires sur notre planète.

Le comportement des principales races zébus du Nord-Cameroun aux techniques modernes d'alimentation intensive apparaît aujourd'hui comme une chose bien connue.
En ce qui concerne les races zébus de l'Adamaoua, les recherches systématiques entreprișes dans ce domaine depuis 1969 à Wakwa, ont établi les excellentes dispositions de notre bétail à l'engraissement.

Diverses rations ont été essayées, divers niveaux de production expérimentés.

Des croisements améliorateurs faisant intervenir par insémination artificielle des races bovines françaises sont expérimentés chez nous depuis 5 ans, en vue de produire des Métis Taurins précoces ou semiprécoces.

L'étude des pãturages de l'Adamaoua et des possibilités fourragères des plantes indigènes ou introduites a donné lieu à la mise au point de techniques intensives de production herbagères et fourragères, dont certaines sont passées au stade de la vulgarisation.

L'alimentation intensive de finition tout comme l'alimentation de soutien en saison defavorable ne soulèvent plus qu'un seul problème et non des moindres : c'est celui de l'indispensable complément, le complément qu'il faut pour donner le «cotip de pouce", ou pour "faire passer la mauvaise saison ».

Classiquernent et d'une façon générale, les systèmes mondiaux d'alimentation du bétail sont fondés sur la distribution d'aliments à haute valeur énergé- 
tique, utilisés seuls ou en association, pour compenser mutuellement d'éventuels déficits. Parmi ces aliments, les céréales constituent l'élément de base, avec les tubercules pour la confection des concentrés qui font si cruellement défaut à notre élevage, ctant donné le faible niveau de production de notre agriculture qui couvre tout juste les besoins alimentaires des populations.

Il est hors de doute qu'il faudrait des décennies pour que cette agriculture atteigne un stade de production autorisant des excédents en céréales et tubercules importants et transférables pour l'engraissement des animaux.

Or les besoins en viande sont pressants : nous ne pouvons pas attendre aussi longtemps.

Il est donc urgent de connaitre rapidement le potentiel de nos régions en matière de cultures industrielles dont la production, transformée sur place, est susceptible de fournir à notre élevage des sousproduits de haute valeur alimentajre.

\section{II. - PROBLEMES POSES PAR LE CHOIX DES ALIMENTS DESTINES A L'ENGRAISSEMENT DES ANIMAUX AU CAMEROUN}

Une foule de produits indigènes sont théoriquement susceptibles d'être doinnés aux animaux comme aliments pour favoriser leur engraissement : les céréales (maïs, mils et sorgho), les tubercules (manioc), les sous-produits agro-industriels (tourtcaux de coton, mélasse de canne à sucre, farines basses de riz) ont été eşayées de façon systématique à Wakwa avec des résultats toujours très bons.

Malheureusement, la plupart de ces produits constituent la base de l'alimentation des populations, dans une agriculture où les problèmes tregents qui se posent en matière de cultures vivrières ne sont pas précisément des problèmes d'abondance, les aléas climatiques et le caractère archaïque de l'exploitation perpétuant une situation de disette chronique.

En ce qui concerne les sous-produits industriels, ils sont :

- soit peu abondants, ce qui résulte du faible niveau de production actuel : c'est le cas du tourteau du coton; la situation est caractérisée ici par une incertitude permanente et par la trop grande fréquence des périodes de pénurie,

- soit peu ou pas valorișés par le bétail : c'est le cas des farines basses de riz de Yagoua et de la mélasse de Mbandjock, ce qui résulte des distances importantes qui séparent les zones de production de celles de consommation, laissant ainsi inexploités des aliments de haute valeur énergétique.

\section{III. - INCIDENCE DE L'IMPLANTATION ET DE LA DIFFUSION DES CULTURES INDUS TRIELLES SUR L'ALIMENTATION DU BETAIL AU CAMEROUN}

L'absence d'une production indigène importante de céréales et de tubercules ne constitue donc pas le seul facteur qui parait limiter dans notre pays le développement de la production intensive de viande.

Une mauvaise utilisation des sous-produits industriels pour les uns, une production insuffisante pour les autres représentent aujourd'hui les facteurs essentiels qui limitent ce développement.

En effet, les céréales et les tubercules ne représentent pas l'unique source d'énergie convertible en protéines animales, constatation qui donne aujour- d'hui raison à W. DAVIES, Président d'honneur aujourd'hui décédé, de la Fondation Européenne des herbages lorsqu'il soutenait, défendant une toute autre cause que c'était une erreur de nourrir le bétail avec « des concentrés de céréales ». Des études récentes qui se classent parmi les plus importantes de la décennie (T.-R. PRESTON à Cuba) confirmées par divers essais au laboratoire (Philippe LHOSTE et collaborateurs à Wakwa), ont apporté la preuve que le zébu peut être engraissé par d'autres sources énergétiques (mélasse) tirant profit de sa faculté d'utiliser l'azote non protéique (urée à Cuba) : ou alors en corrigeant le déficit protéique de cette denrée par le tourteau de coton (Wakwa).

Par aillcurs, des expériences d'alimentation menćes à Wakwa et sur le terrain, ont établi que les races zébus du Nord-Cameroun pouvaient rentabiliser des rations à base de tourteatix et de graine de coton avec des bénéfices parfois considérables.

Des essais d'utilisation des issues de rizerie provenant de Yagoua, dans les rations d'engraissement des zébus de l'Ádamaoua ont démarré il y a deux ans et les résultats paraissent prontetteurs à tous les égards.

L'existence d'une gamme variée de cultures agroindustrielles parfaitement adaptées au Cameroun, constitue pour l'alimentation du bétail de notre pays u1h atout. L'exploitation rationnelle de ces cultures et leur transformation sur place fournira à notre cheptel des quantités importantes de sous-produits qui constituent des aliments riches susceptibles de faire passer notre élevage du stade de la cueillette à celuii de "I'élevage activité économique".

\section{1) Culture cotonnière}

La culture * cotonnière ", locomotive de l'économie du Nord, est inscrite comme opération stratégique dans le III ${ }^{*}$ Plan Quinquennal, les objectifs en 1976 étant la production de 120000 tonnes de coton sur 130000 hectares soit un rendement moyen de $920 \mathrm{~kg} /$ hectare. Avec ul rendement à l'égrenage de l'ordre de 36,6 p. 100 , cette production peut permettre d'espérer 43920 tonnes de graine en 1976.

Le taux d'extraction variant entre 16 et 19 p. 100 , si nous prenons comme chiffre moyen 18 p. 100 , on peut ainsi escompter 36014 tonnes de tourteaux de valeur alimentaire estimée à I UF et 350 gramunes de MPD au $\mathrm{kg}$ de matières sèches.

Avec un indice de consomtation moyen de 8 à 9 (jeunes animaux) : nombre d'UF nécessaires pour la production du $\mathrm{kg}$ de viande, c'est 4502 tonnes de viande supplémentaire qui auraient pu être produits en 1976 en tirant profit du coton, dans l'hypothèse que l'ensemble de la production est écoulée à l'intérieur : cette production représente notre déficit en 1969 estimé à 5800 tonnes de viande.

\section{2) Culture de la canne à sucre}

I] existe au Cameroun une industrie sucrière en développement. En 1970-1971, la S.O.S.U.C.A.M. pro duisait à Mbandjock entre 10000 et 42000 tonnes de sucre sur 3200 hectares (3750/hectare).

L'objectif de production du complexe est de 20000 tonnes de sucre pour 1975-1976. D'autre part la consommation du sucre en année terminale du III ${ }^{\mathrm{E}}$ Plan Quinquennal se situera probablement entre 40 et 50000 tonnes.

La production nationale représente donc le $1 / 4$ de la consommation et quand l'objectif fixé sera atteint, la production ne couvrira que la moitié seulement de la consommation. C'est pourquoi le III Plan Quinquennal prévoit, soit un agrandissement plus important à Ḿbandjock, soit la création d'un nouveau complexe. 
Or, il n'est pas un seul village du Nord-Cameroun où ne se pratique la culture familiale de canne à sucre. D'autre part, le coût de transport de la mélasse produite à Mbandjock rend prohibitive l'utilisation de cette denrée au Nord, denrée qui, comme le montre PRESTON à Cuba, n'est rentabilisée que si elle est utilisée au lieu de la production (distribution "ad libitum »).

Un projet de culture de la canne à sucre dans le nord entre donc bien dans l'objectif du III" Plan Quinquennal et se justifie par l'importance de la tradition de cette culture, sa rentabilité étant garantie par la possibilité d'exploitation de la mélasse par les animaux de la province.

Il ne resterait qu'à connaître par des études préalables, si cette canne à sucre produite localement peut être valablement utilișée à des fins industrielles.

Ce projet fera l'objet d'une opération intégrée (Aménagement du nord-est Bénoué, à Garoua). Avec l'augmentation de la capaçité de production à Mbandjock, il permettra par la création d'un point de production de mélasse au Nord-Cameroun, l'utilisation rationnelle de cette denrée pondéreuse pour laquelle le transport limite la rentabilité.

Dans le cadre du Plan Viande au Cameroun, la mélasse de Mbandjock trouvera une excellente utilisation dans les ranches voisins de Ndokayo (Ndokayo 1 et Ndokayo 2).

\section{3) Culture du ble}

De cette culture industrielle, l'élevage tirera pour l'alimentation du bétail outre les pailles, un avantage pastoral certain résultant des méthodes culturales appropriées, notamment la méthode des cultures dérobées dites «avec plante de couverture" celle-ci pouvant être une plante comestible ou fourragère exploitable

Au moulin, les sons qui forment la moitié des issus (petits sons, sons moyens, gros sons : sons trois cases) ont eu de tout temps une grande valeur auprès des éleveurs; l'abondance de la cellulose fait qu'il y a une bonne utilisation directe chez les ruminants. Les protides sont abondants et de haute qualité, les lipides aussi ; la teneur en sels est généralement élevée, sauf le calcium : la valeur énergétique du produit est estimée à 0,7 UF.

Des essais de culture de blé ont été conduits depuis trois ans dans le Nord-Cameroun par la Société Camerounaise de Minoterie (S.C.M.) Des cultures ont été implantées dans deux localités :

- Garoua ;

- Ngaoundéré (Wakwa).

Les résultats ont ćté assez vite prometteurs à Ngaoundéré, tandis qu'ils se soldaient par un échec à Garoua.

En ce qui concerne Ngaoundéré, il convient de signaler la spontanéité avec laquelle la nature a répondu de facon acceptable à cette culture. De fait, le site de l'Adamaoua est climatiquement favorable.

Il en est résulté une tradition de culture de blé dur, vieille de plus de 20 ans à Wassande, dans un village proche de Ngaoundéré à 80 kilomètres de cette localité, avec des rendements très bons de l'avis des experts.

Les essais entrepris à Wakwa perdent de ce fait leur caractère pionnier. Les avantages pastoraux indéniables susceptibles d'être tirés de cette culture industrielle, militent en faveur de son démarrage et de son extension à grande échelle pour la mise en route d'un grand moulin à Ngaoundéré. Toutefois ce problème se pose de deux façons différentes suivant qu'il s'agit de zones déjà exploitées (périmètre de Wassande) ou de zones vierges.

\subsection{Cas au périmètre Wassande}

L'occasion unique nous est donnée ici pour faire l'expérience d'une association culturéélevage. Tenant compte du fait que la vocation des populations est essentiellement pastorale, l'encadrement avec des moyens suffisants pourrait se faire dans le sens d'une promotion d'exploitations familiales d'élevage et de cultures, le mode de culture indiqué étant la culture dérobée avec plantes fourragères de couverture : Stylosanthes gracilis trouve sur ces sols une écologie exceptionnelle, quand on en juge par l'extraordinaire réussite des champs de vulgarisation que nous avons exécutés dans les villages voisins de Nyambaka.

\subsection{Cas de culture sur grandes superficies}

Ce sont elles qui seraient le vrai support du grand moulin de Ngaoundéré. Le terrain ne manque pas; la physionomie des sols de Wakwa se retrouve un peu partout en Adamaoua, l'altitude aussi.

\section{IV. - DEFINITION D'UNE POLITIQUE DE DEVELOPPEMENT EN MATIERE DE LA PRO- DUCTION INTENSIVE DE VIANDE}

L'orientation prise au III ${ }^{\text {Plan }}$ Quinquennal de Développement Economique et Social du Cameroun, en matière d'élevage bovin est donc nettement vers une production intensive.

Le spectre d'un déficit de viande au Cameroun est à juste titre insupportable pour un pays actuellement exportateur.

Les dispositious envisagées, devant permettre dans les dix ans de faire honorablement face à la conjoncture qui se dessine tendent toutes vers une meilleure utilisation du disponible local, c'est-à-dire de toutes les sources de protéines et d'énergie disponibles donc transférables pour l'engraissement des animaux au prix de revient le plus bas.

Tenant compte du fait que, qui dit alimentation des ruminants, dit fourrages avant tout au sens large du mot, la place des fourrages à pâturer ou à faucher dans notre politique de développement des productions animales est privilégiée : la vulgarisation chez certains "éleveurs d'avant-garde" des méthodes intensives d'exploitation des pâturages est une des préoccupations du gouvernement.

Notre politique pour la promotion de l'élevage moderne au Cameroun peut dès lors se résumer da la façon suivante :

- politique de l'utilisation des sous-produits,

- politique d'encadrement et d'éducation : vulgarisation intensive des techniques rationnelles d'alimentation du bétail.

1) Politique de l'utilisation des sous-produits industriels

\subsection{Au niveau de la production}

Elle vise à améliorer l'alimentation du cheptel d'une part en élargissant l'éventail des sous-produits locaux susceptibles d'une transformation économique en protéines animales, d'autre part en favorisant une meilleure utilisation de ces sous-produits par une implantation judicieuse des exploitations agro-industrielles et l'intégration à la base du cheptel aux « opérations agricoles ",

L'intégration du cheptel à l'exploitation agro-indus trielle garantira l'écoulement des sous-produits qui ainsi rentabilisés, participeront effectivement à 
l'amortissement du complexe, avec effet sur l'atténuation des écarts des cours.

\subsection{Au niveau de la commercialisation}

Compte tenu de notre faible production de céréales et de tubercules, notre politique tendra à privilégier la consommation intérieure des sous-produits agro-industriels en limitant la part clestinée à l'exploitation par les sociétés agro-industrielles.

Les exportations des sous-produits agro-industriels, source de devises pour l'amortissement du complexe se justifiaient à un moment où ils ne donnaient lieu a aucune utilisation locale. 11 devient aujourd'hui inconcevable que Ie Cameroun dont le cheptel meurt de faim, continue à exporter vers les pays riches ces sources de protéines et d'énergie dont il a besoin pour la réalisation de ses objectufs fondamentaux.

Une péréquation pourrait être étudiée avec les sociétés agro-industreilles opérant au Cameroun.

\section{2) Polilique de vulgarisation des techniques} rationnelles d'alimentation du bétail

C'est une politique d'éducation des éleveurs. L'animation pastorale, support de cette éducation, fonctionne dans les grandes zones d'élevage depuis le début du III Plan Quinquennal avec les movens suffisants.

En ce qui concerne l'Adamaoua, le Centre de Recherches Zootechniques et Fourragères de l'I.E.M.V.T. au Cameroun est doublé à Wakwa d'une station de production, chargée de vulgariser auprès des éleveurs camerounais les techniques d'alimentation et les schémas de production mis au point par la recherche.

Les campagnes de complémentation collectives des troupeaux traditionnels peodant la saison sèche, organisées au niveau des villages et groupes de villages, ont démarré l'année dernière et seront exécutées de façon systématique tous les ans.

Le complément alimentaire distribué aujourd'hui cst. le tourteau de coton sur la base de 500 grammes/ tête/jour. Les animaux bénéficiant de ce régime sont les jeunes sevrés, les femelles suitées défavorisées par un gros veat à la mamelle, les génisses dans le but de réduire l'âge au premier veau, et enfin les bouvillons de 3 à 4 ans en vue de les maintenir en état pendant la saison sèche, pour une commercialisation. aù début des pluies.

En ce qui concerne l'embouche, des moyens suffisants sont mis en ceuvre en Adamaoua pour amener un certain nombre de commerçants à bétail à finir en saison défavorable, par une alimentation semiintensive fondée sur la distribution sur savane riche, avec réserves fourragères, d'un complément alimentaire.

Des prairies individuelles de 4 à 5 hectares de Stylosanthes gracilis ont été cultivées l'anjuée dernière pour des éleveurs d'avant-garde, à titre de démonstration.

Chaque prairie artificlelle était doublée d'une prairie d'herbe de savane de 5 à 10 hectares aménagé spécialement pour la fauche : wne haie de trois lignes de barbelés limitant largement l'enscmble, ménageait à l'extérieur des prairies à faucher, une dizaine d'hectares de parcours de bonne qualité à pâturêr pendant la saison sèche.

La mise au point de ce système de vulgarisation n'a été rendue possible que par la solution du problème de trésorerie des éleveurs et des commerçants à bétail. La possibilité d'octroi des prêts de campagne comparables à ceux pratiqués autx agriculteurs est en étude par un organisme spécialisé du gouvernement : le F.O.N.A.D.E.R. (Fonds National de Développement Rural), qui pourra ainsi préfinancer l'achat du tourteau et le stocker dans des entrepôts villageois, de mềme que le sel, le natron, le matériel agricole.

En ce qui concerne ce secteur intensif en gestation, un certain avenir semble destiné à l'association coopérative des "emboucbeurs" au nivcau du village ou du groupe de villages, disposant :

- d'un magasin d'entreposage des denrées alimentaires (tourteau, farines basses...) et des produits de première nécessitć : sel, natron, pompes pour détiquage, insecticidies,

- d'un parc de matériel agricote.

Dès lors il n'y aurait plus lieu de multiplier les petites prairies individuelles; il serait envisagé au contraire, la création dans les principales zones d'élevage, de prairics à grandes surfaces destinées à lourmir le fourrage (en bottes pressées de $100 \mathrm{~kg}$ ) à tous les cmboucheurs de la localité.

De telles prairies ont en effet de nombreux avantages :

- possibilité d'atteindre un grand nonbre d'éleveurs, au lieu du petit nombre actuellement. Ainsi, pas de particularisme, une saine émulation entre les éleveurs,

- prix de revient moins élevé que la multiplication de petites prairies individuelles qu'il faudrail continuellement entretenir à grands frais,

- suppression de nombreux déplacements : tout le matériel étant sur place pour l'eutretien de la prairie, la coupe du fourrage, le stockage et la distribution,

- garantie d'un bon séchage assuré pour une conservation d'une durée illimitée dès le début de Ia saison sèche,

- transport facile cn bottes pressées, encombrement réduit,

- facilité de stockae du fourrage,

- facilité de distribution : bottes de $100 \mathrm{~kg}$ (5 kg par bête : 1 botte pour 20 betes),

- pas de gaspillage, aucune perte de fourrage, ni sur le terrain, ni à la distribution.

Un projet est en préparation et sera présenté au F.O.N.A.D.E.R.; le matériel nécessaire envisagé est le suivant pour un groupement d'emboucheurs :

- Tracteur ;

- Charrue:

- Herse en branchages;

- Fauchcuses;

- Rateleuses;

- Boudineuses;

- Presse à fourrage (le choix de cette presse doit etre étudié avec attention : c'est la machine la plus inportante de l'entreprise);

- Hangars de stockage du fourrage ;

- Hangars de stockage du tourteau;

- Sel et natron;

- Matériel de clôture;

- Véhicules de service;

- Camions pour la distribution du fourrage et du tourteau ;

- Logements du personmel, etc.

\section{V. - MISE AU POINT D'UN SYSTEME D'EN- GRAISSEMENT DU BETAIL FONDE SUR LA DISTRIBUTION D'UN COMPLEMENT ALIMEN- TAIRE : LE TOURTEAU DE COTON}

L'orientation prise par l'agriculture camerounaise dans le cadre de la "Revolution Verte" et qui se concrétise aujourd'hui par la création par le gouvernement des missions de développernent des cultures 
industrielles (blé au nord, riz dans la plaine des Mboums, canne à sucre, etc.), doit pouvoir mettre à la disposition des éleveurs dans les 10 ans à venir, une gamme variée de sous-produits agro-industriels pour l'aimentation de leurs animaux.

Il appartiendra aux techniciens de mettre au point des schémas d'alimentation simples et vulgarisables susceptibles de faire bénéficier au mieux ces sousproduits à notre élevage.

Ainsi, dans le contexte agro-industriel du Nord Cameroun, dominé pour le moment par la culture du coton, il est apparu particulièrement intéresșant de mettre au point et de vulgariser un système d'embouche simple fondé sur la finition à contre-saison des jeunes bœufs de 3 à 4 ans, par la distribution du tourteau de coton.

L'utilisation du tourteau se justifie ainsi par :

- la simplicité : n'exige aucune préparation,

- le prix : le tourteau produit au nord reste deptis quelques anneés la denrée la moins chère au NordCameroun pour l'alimentation du bétail.

1) Bases expérimentales du système vulgarisé en Adamaoua

Le protocole initial a été mis sur pied à Wakwa en 1969 par Philippe LHOSTE et Robert DUMAS. Il fut repris à la Station Fourragère de Wakwa par Jacques PIOT en 1971.

Ce schéma de finition basé sur la complémentation sur savane naturelle ayant acquis par sa simplicitó notre faveur, fut repris par nous-mêmes en vraic grandeur sur le terrain au cours d'un essai merie pendant la saison sèche 1972-1973 dans le village de Ngaoundéré, dans les conditions du milieu. Cet essai tirait son intérêt de son caractère pionnier : en effet, il n'avait pas encore été exćcuté auparavant.

Les résultats obtenus dans les conditions aussi dures, dominées par l'ambiance de saișon sèche (feux de brousse), autorisaient d'espérer des performances pondérales très correctes pour peu que l'on modifie le milieu : qualité du pâturage, réserves fourragères, facilité de l'abreuvement.

\subsection{Les aninatux}

Un lot de 10 boufs zébus de race locale, d'âge compris entre 3 et 4 ans, intégrés dans un troupeau intensif de 50 têtes, sont isolés une heure par jour pendant 85 jours pour recevoir le complément alimentaire (tourteau de cotori) distribué dans une auge collective en bois de 6 mètres, placée dans une petite enceinte clôturée avec une auge à pierre à lćcher.

Pareils animaux sont élevés 2 à 3 ans de plus pour être commercialisés, comme nous l'avons vu, à 5-6 ans pour un gain pondéral variant de 50 à $100 \mathrm{~kg}$.

Les résultats que nous rapportons dans les tableaux ci-dessous sont relatifs à 9 bceufs, les performances du $10^{\circ}$ n'ont pas été prises en considération pour des raisons de pathologie.

\subsection{Consommation}

Les animaux sont entretenus dans les conditions extensives de saison sèche avec quelques pailles sur pied sur brûlis.

La récolte est faible, les aliments peut-être riches, mais une énorme énergie de récolte est dépensée. Si pendant les premiers jours, le milieu équilibre dans une bonne mesure les besoins d'entretien des animaux, très vite tout est consommé et la pratique nous enseigne qu'il y a par la suite, une perte rapide de poids si un complément alimentaire n'est pas dispensé aux animaux.

Rappelons également que, concernant les exigences alimentaires des animaux, les normes « Boudet
Rivière " pour les animautx de $350 \mathrm{~kg}$ à l'entretien et de (très) petits déplacements, ce qui n'est pas le cas ici, donnent environ 200 grammes par jour de M.P.D.

Nous pouvons donc faire comme si tout l'azote de la ration devait être apporté par le complément. C'est une grosse approximation, mais elle nous paraît suffisante.

La valeur alimentaire du tourteau de coton est estimée à I UF et 350 grammes de M.P.D. au $\mathrm{kg}$ de matière sèche.

Nous donnons ci-dessous les quantités moyennes consommées par tête et par jour :

$\begin{array}{ll}-1^{\mathrm{rc}} \text { à } 2^{\circ} \text { semaine } \ldots \ldots \ldots \ldots \ldots \ldots \ldots \ldots & 1,0 \mathrm{~kg} \\ -3^{\circ} \text { semaine } \ldots \ldots \ldots \ldots \ldots \ldots \ldots \ldots \ldots \ldots & 1,5 \mathrm{~kg} \\ -6^{\circ} \text { semaine } \ldots \ldots \ldots \ldots \ldots \ldots \ldots \ldots & 2,0 \mathrm{~kg} \\ -7^{\circ} \text { à } 12^{\circ} \text { semaine } \ldots \ldots \ldots \ldots \ldots \ldots \ldots & 3,0 \mathrm{~kg}\end{array}$

- Consommation moyenne par tête durant la période de complémentation $\ldots \ldots \ldots \ldots \ldots \ldots \ldots \ldots \ldots, 181,0 \mathrm{~kg}$

\subsection{Résultats}

\subsubsection{Evolution pondérale}

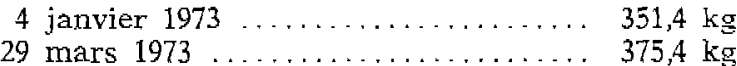

Croît moyen par lête au cours de

3 mois ..................... 24,0 kg

Variation de poids : gain de poids jour-

nalier moyen en $\mathrm{g} / \mathrm{j} \ldots \ldots \ldots \ldots \ldots$......... 282,3 $\mathrm{g}$

Ce tableau montre que le but recherché a été largement atteint puisque, malgré les fluctuations de régime, le poids du bétail s'est accru pour atteindre en fin de complémentation les $6,82 \mathrm{p}$. 100 du poids de départ. PIOT a obtenu à Wakwa 8,5 p. 100 pour les arimaux de même race et de même poids, avec, il est vrai, un régime moius poussé, mais en contrepartie sur des parcs riches en refus de la saison des pluies précédentes et un matériel végétal disponible, compté comme faisant environ 0,4 UF et 6 grammes de MPD au kîlogramme de MS. Quant à LHOSTE, il a obtenu avec des animaux identiques des variations de poids de plus de $47,9 \mathrm{~kg}$ sur savane, graines de coton à volonté, minéraux et une réserve de foin de saison sèche.

\subsection{Données économiques}

a) Coût de l'opération

Les dépenses d'installation sont minimes, donc négligeables : il n'y a aucun investissement d'infrastructure. Voici les autres postes de dépenses :

\section{Alimentation :}

- $181 \mathrm{~kg} /$ tête pour 9 boufș à $19 \mathrm{~F} / \mathrm{kg}$. - $30951 \mathrm{~F}$

- 1 auge de complémentation ........ $4295 \mathrm{~F}$

- 1 pierre à lécher ............. $3510 \mathrm{~F}$

Coût total de l'alimentation pour les

9 bœufs .................... $38756 \mathrm{~F}$ Soit par un boeuf ............. $4306 \mathrm{~F}$

b) Plus-value apportée d̀ l'animal et calcul du bénéfice :

- Valeur d'achat des animaux (pièce) .

- Prix de vente au crochet après trois mois de complémentation :

- Poids moyen de carcasse pantelante: $186 \mathrm{~kg}$ à $180 \mathrm{~F} / \mathrm{kg} \ldots \ldots \ldots \ldots \ldots \ldots, 33480 \mathrm{~F}$

- Plus-value : $33480 \mathrm{~F}$ - $18000 \mathrm{~F} \ldots$.. $15480 \mathrm{~F}$

c) Détermination du bénéfice net

Plus-value/bøuf ................... $15480 \mathrm{~F}$

Coût bouf ..................... $4306 \mathrm{~F}$

Bénéfice net/boeuf :

$15480-4306 \mathrm{~F} \ldots \ldots \ldots \ldots \ldots \ldots 11174 \mathrm{~F}$ 


\subsubsection{La leçon à tirer}

En effet :

Ces résultats paraissent riches d'enșeignements, ils prouvent :

1) que dans les plus dures conditions de l'étevage extensif en saison défavorable, le zébu peul de l'Adamaoua peut augmenter son poids de façon non négligeable, si l'indispensable apport protéique lui est assuré,

2) que les performances pondérales, et ceç découle en ligne directe de ce qui précède, peuvent être nettement améliorées par un équilibre de la ration.

\section{2) Principe du protocole vulgarisé}

Dans le protocole vulgarisé, il s'agit d'amener des conmerçants et des agriculteurs-éleveurs à regrouper un certain nombre de bceufs non finis de 3 à 4 ans et destinés à la boucherie sur un pâturage déterminé. Ces animaux sont gardés un certain temps ( 1 an : une saison des pluies et une saison sèche) et reçoivent aut moment le plus favorable à leur croissance, une ration de complémentation.
Le schéma retenu est une finition en saison sèche sur savane naturelle avec réserves fourragères.

Conduite du troupeau : node d'élevage

Ce schéma de production de viande comporte deux régimes :

— Régime extensif de mars à décembre :

- Sur pâturage, plus légère complémentation en saison des pluies;

- Apport minéral à volonté (pierre à lécher).

- Régime semi-intensif de janvier à mars :

- Sur pâturage naturel assez vaste (1 hectare par animal), clôturé, en bon état et comportant un point d'eau à proximité de la zone d'alimentation

- Apport minéral à volonté :

- Réserves fourragères.

\section{3) Durée de l'engraissement}

La durée de l'engraissement est de trois mois. L'objectif est la commercialisation de carcasses de qualité au retour des pluies, c'est-à-dire à une période de pénurie qualitative et quantitative du système de production traditionnel.

4) Plan indicatif de rationnement

\begin{tabular}{|c|c|c|}
\hline date approximetive & type d’alimentation & rations et quantité de tourteau en kg/sour/têțe \\
\hline $\begin{array}{l}\text { de mars-avril à la } \\
\text { premiere quinzaine de } \\
\text { dêcembre. }\end{array}$ & rëgime extensif & 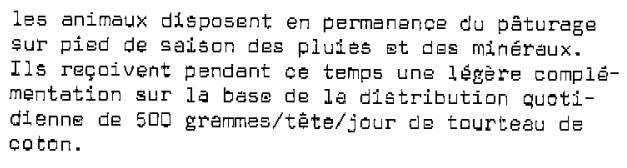 \\
\hline $\begin{array}{l}\text { Zème quinzoine de } \\
\text { décembre au ler } \\
\text { janvier de I'année } \\
\text { suivente. }\end{array}$ & I’égimb semi-intensif & 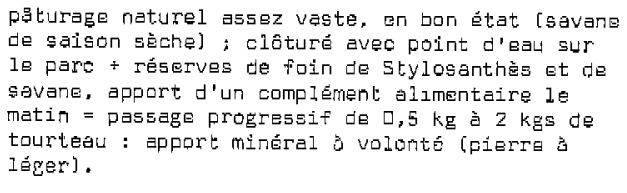 \\
\hline de janvier à mars & semi-inteneif & $\begin{array}{l}\text { même chose que plus haut : la quantité do tourtoal } \\
\text { distribute par tête et par jour }=2 \text { ou } 3 \mathrm{~kg} \text { ge } \\
\text { selun le cos. }\end{array}$ \\
\hline
\end{tabular}

\section{CONCLUSION}

Dans la course au développement économique et social dans laquelle le Canneroun est engagé, l'accroissement de la production agricole au sens large du terme apparaît comme l'un des premiers buts, sinon le premier à atteindre.

La réalisation des cultures industrielles permet, outre l'obtention des devises grâce aux exportations (café, cacao...), la création d'industries locales de transformation (huileries, sucreries, meuneries...) dont les sous-produits constituent des aliments de grande valeur pour les animaux domestiques.

\section{BIBLIOGRAPHIE}

1. CRAPLET, L'alimentation du bétail (1950).

2. BOUDET (G.) et RIVIERE (R.), Emploi pratique des analyses foutragères pour l'appréciation des pâturages tropicaux. Rev. Elev. Méd. vét. Pays trop., Tome XXI Nouvelle Série), No $2,1968$.
3. MONGODIN (B.) et RIVIERE (R.), Valeurs bromato logiques de 150 aliments de J'Ouest Africain, I.E.M.V.T janvier 1965 .

4. I.E.M.V.T. WAKWA, Bilan annuel pour 1972/1973.

5. Ministère de l'Elevage et des Industrics Aninales, Programme Viande au Cameroun.

6. IIF Plan de Développement Economique et Social, Conmissions Régionales de Planification pour le NordCameroun, 18,19 et 20 juin 1970.

7. EL HADJ AHMADOU AHIDJO, Président de la République Unie du Cameroun : Vers la Révolution Verte.

8. Projet de culture du blé et d'un moulin à blé au NordCameroun, S.O.M.E.D.I.A. - S.C.M., Grand Mouliz de Paris, 1972 .

9. PRESTON (T.-R.), L'engraissement des bovins de boucherie à la mélasse dans les régions tropicales Revue Mondiale de Zootechnie, $\mathrm{N}^{\circ} 1$, 1972 .

10. Rapport annuel 1972 de l'Inspection Provinciale d'Agriculture et d'Animation Rurale pour le Nord-Cameroun.

11. LHOSTE (Ph.), PIERSON (J.-N.), GINISTY (L.), Essai de finition de boeufs zébus avec utilisation maximale de mélasse : I.E.M.V.T. Wakwa, août 1973. 